\title{
Intervenção precoce na comunicação pais-bebê com deficiência visual ${ }^{1}$
}

\author{
Early intervention in parent-baby interaction \\ in a context of visual impairment
}

\author{
Carla Meira KREUTZZ 2,3 \\ Cleonice Alves BOSA B $^{2,4}$
}

\begin{abstract}
Resumo
A deficiência visual congênita, que pode levar à cegueira, tem sido vista como risco para a interação mãe-bebê e para o desenvolvimento psicológico da criança. O bebê que nasce com uma deficiência visual acentuada tem uma relação diferenciada com o mundo, sendo que outras vias de comunicação e percepção terão de ser ativadas para que ele alcance o desenvolvimento. Os pais são de extrema importância para o desenvolvimento desse bebê, o qual, mais do que nunca, necessita deles para entrar em contato com seu ambiente. Nesse sentido, o objetivo deste artigo é revisar a produção científica na área de intervenção precoce com pais e bebês que apresentam deficiência visual congênita. Para isto, identificam-se controvérsias teóricas acerca do desenvolvimento destes bebês, aspectos da intervenção que favorecem a interação pais-bebê e dificuldades metodológicas dos estudos nesta área.
\end{abstract}

Unitermos: Estimulação precoce. Família. Portadores de deficiência visual.

\begin{abstract}
The congenitally impaired vision that can result in blindness is a risk factor for mother-baby interaction and for the child's psychological development. The visually impaired or blind baby has a different interaction with the world, so alternative ways of communication and perception must be activated to allow for development. Thus, the parents have an important role in their child's development, as the baby needs them more than ever to begin making contact with the outside world. The aim of this article is to review the scientific production in the area of early intervention with blind babies and their parents. To this end, we have identified theoretical discussions concerning the development of blind babies, early intervention issues to help parent-baby interaction and methodological difficulties with these studies.
\end{abstract}

Uniterms: Early intervention. Family. Visually impaired individuals.

Deficiência Visual (DV) é um termo utilizado para designar os impedimentos de origem orgânica relacionados a doenças oculares que podem dificultar o funcionamento da visão ou levar à ausência desta, com ou sem a percepção de luz, podendo ser herdada ou adquirida (Batista \& Enumo, 2000). Dados precisos

\section{$\operatorname{cov}$}

1 Artigo elaborado a partir do projeto de tese de C.M. KREUTZ, realizado sob supervisão de Cleonice Alves Bosa, apresentado ao Programa de Pós-Graduação em Psicologia, Universidade Federal do Rio Grande do Sul. 2005.

2 Universidade Federal do Rio Grande do Sul, Instituto de Psicologia, Programa de Pós-Graduação em Psicologia. R. Ramiro Barcelos, 2600, 90035-003, Porto Alegre, RS, Brasil. Correspondência para/Correspondence to: C. M. KREUTZ.E-mail: <carla.kreutz@gmail.com>.

3 Universidade Luterana do Brasil, Curso de Psicologia. Porto Alegre, RS, Brasil.

- Núcleo de Estudos e Pesquisas em Transtornos do Desenvolvimento. Porto Alegre, RS, Brasil. 
quanto à prevalência da deficiência visual profunda são pouco encontrados na literatura, ficando entre 0,3 e 1,5 em cada 1 mil crianças. Tende a ser maior nos países com um nível socioeconômico mais baixo (Gilbert \& Foster, 2001).

\section{A DV e a interação pais-bebê: implicações para intervenção precoce}

Estudos com bebês nos primeiros meses de vida com desenvolvimento típico têm fornecido evidências de que as crianças têm um maior interesse por aspectos do ser humano do que por estímulos equivalentes, assim como um repertório de capacidades sociais, as quais têm grande potencial de comunicação, quais sejam, o choro, o sorriso e a imitação (Messer, 1994), o que auxilia na interação social da criança com os indivíduos em especial. Através desses sinais, o bebê pode dar início às trocas comunicativas com seus cuidadores, as quais constituem os pilares do desenvolvimento da comunicação.

A fase diádica do desenvolvimento da comunicação corresponde à interação que ocorre entre cuidador e criança, primordialmente nas interações face a face. Segue-se a fase triádica do desenvolvimento, na qual a criança já tem uma noção mais clara sobre si e sobre o outro e consegue compartilhar um evento ou objeto externo com alguém (Bosa, 2002). A criança já compreende referências sociais, ou seja, informações emocionais transmitidas pelas expressões faciais dos adultos (Pérez-Pereira \& Contí-Ramsden, 1999).

Nesse contexto, a visão, como um dos sentidos que possibilita a ligação com o mundo social, tem grande importância para que o indivíduo possa interagir adequadamente com os que o rodeiam. A visão assegura que as crianças se orientem para elementos do ambiente que são relevantes para o desenvolvimento e para a sobrevivência, como a orientação para faces humanas (Adelson \& Fraiberg, 1974; Pérez-Pereira \& Contí-Ramsden, 1999). A criança cega ou com DV severa é privada de uma série dessas oportunidades; assim, as respostas contingentes às expressões do cuidador podem ficar reduzidas (Pérez-Pereira \& Contí-Ramsden, 1999). Os estímulos auditivos e táteis são vistos como alternativas de exploração e interação para as crianças que têm DV, embora o estímulo auditivo tenha menor potencial motivacional para que a criança vá ao encontro dos objetos do que o visual (Adelson \& Fraiberg, 1974).

É fundamental uma estimulação adequada por parte dos parceiros de interação do bebê, os quais necessitam ser sensíveis a distintos sinais comunicativos do lactente para propiciarem um ambiente estimulante e alcançarem uma interação sincrônica. No entanto, o cuidador pode se sentir desestimulado na interação, uma vez que não recebe as respostas habituais de um bebê. Pelo contrário, as crianças cegas reagem frequentemente por meio do silêncio e apresentam uma menor movimentação quando ouvem seus cuidadores falarem ou expressarem outros comportamentos comunicativos (Als, Tronick \& Brazelton, 1980). Desta forma, os ciclos de interação podem ser prejudicados e, consequentemente, também o desenvolvimento da comunicação.

Estudos têm apontado que a falta de contato olho a olho e de atenção visual em crianças cegas resulta em diminuição da atenção visual e responsividade vocal da mãe (Rogers \& Puchalsky, 1984; Rowland, 1984; Warren, 1994). Como o olhar é uma pista importante para que as mães possam responder adequadamente à orientação inata de seus bebês e possam criar ciclos interativos de comportamentos e afeto sincronizados, a cegueira pode exigir muito dos pais em termos de percepção de necessidades e comunicação. Para Perez-Pereira e Contí-Ramsden (1999), a deficiência visual não traz prejuízos para o desenvolvimento da criança por si só, mas isso pode ocorrer caso não sejam criadas vias alternativas para o desenvolvimento das diversas capacidades da criança. Portanto, profissionais da saúde têm amplo campo para auxiliar os pais na descoberta dessas vias. Parece haver diferenças na interação das mães com bebês com DV. No entanto, muitas vezes essas diferenças podem indicar um aspecto positivo para o desenvolvimento da criança.

Uma pesquisa realizada por Kekelis e Andersen (1984) examinou os efeitos da DV na interação pais-criança, objetivando investigar como os pais utilizam a linguagem com seus filhos com DV e como esta se modifica com o desenvolvimento do filho, bem como compreender a relação entre os inputs dos pais e os comportamentos da criança. Os participantes do estudo longitudinal eram seis crianças, acompanhadas dos 16 
aos 36 meses, com graus variados de DV, e duas com visão. Os resultados indicaram que os cuidadores das crianças com DV apresentaram um maior número de proposições diretivas, indicando que os pais tentam encorajar a criança a ter um papel mais ativo na conversação e na exploração do ambiente. No entanto, os pais acabaram por repetir diversas vezes a solicitação à criança, fazendo com que esta se afastasse e se retirasse do tópico, ao invés de atender ao que foi solicitado. Os pais também propiciaram um menor número de descrições, nomeando muito mais os objetos do que os descrevendo, o que é surpreendente, pois se esperaria que os cuidadores descrevessem mais o ambiente para que a criança pudesse melhor explorá-lo. As autoras atribuíram essa falta de descrições à dificuldade dos pais em monitorar a compreensão da criança sobre o que eles estão explicando. Além disto, iniciaram uma porcentagem bem maior de tópicos do que seus filhos, além de enfocarem mais exclusivamente tópicos centrados na criança, ao invés de no ambiente, se comparados aos pais das crianças videntes.

Kekelis e Andersen (1984) afirmam que as proposições diretivas são limitantes por dois motivos. Um deles é que elas não proporcionam à criança um contato com funções mais ricas da linguagem. Além disso, a criança fica com poucas oportunidades de resolver os problemas criativamente, se os outros sempre fazem o que ela solicita. Os pais devem encorajar as crianças a fazerem mais do que solicitar objetos e ações dos outros. É comum que as crianças com DV reclamem por assistência e acreditem que não conseguirão executar determinadas tarefas, porém elas podem adquirir um senso de autoeficácia se forem encorajadas a alcançarem seus próprios objetivos. Para tanto, considera-se importante ressaltar que os pais necessitam, em primeiro lugar, se sentir confiantes nas possibilidades de desenvolvimento de seu filho, o que exige também confiança em si mesmos como pais. Este senso de autoeficácia pode estar prejudicado pelo abalo emocional oriundo da descoberta da deficiência do filho, assim como por uma interação pais-bebê assincrônica. Esses aspectos devem ser foco de intervenção para que o desenvolvimento da criança siga um curso saudável do ponto de vista emocional.

Ainda sobre a relação entre deficiência visual congênita e interação social, no contexto da interação diádica mãe-criança, Souza, Bosa e Hugo (2005) investigaram a presença de condutas do espectro do autismo em crianças portadoras de Deficiência Visual Congênita (DVC) e o estilo interativo materno. Para isso, foram estudadas, comparativamente, oito díades mãe-criança (quatro com deficiência visual congênita severa e quatro com desenvolvimento típico). As crianças de ambos os grupos tinham entre dois e seis anos. Um dos importantes achados das autoras foi que duas das quatro crianças com deficiência visual apresentaram frequências maiores de atenção compartilhada, quando comparadas às crianças com desenvolvimento típico, utilizando modalidades verbais e não verbais (orientar a cabeça ou o corpo em direção ao campo de visão da mãe, entregar ou colocar objeto no campo de visão da mãe). Concluíram que as mães dessas crianças tiveram um papel decisivo para esse resultado. Elas foram mais sensíveis às necessidades da criança ao descreverem as propriedades dos brinquedos, ao buscarem semelhanças entre os brinquedos do laboratório de observação e aqueles que as crianças possuíam em casa e ao utilizarem caminhos alternativos durante a exploração do ambiente, como o tato, por exemplo.

Ainda nesta linha de investigação, Behl, Akers, Boyce e Taylor (1996), em um dos poucos estudos com uma amostra que permitiu uma alta validade estatística, estudaram os padrões interativos de mães de crianças com deficiência visual, comparando-os com os padrões de comportamentos interativos de crianças que enxergavam perfeitamente. Os autores também objetivavam verificar se haveria alguma relação entre o comportamento interativo materno e os diferentes graus de visão da criança. Para tanto, participaram da pesquisa 31 crianças com diferentes níveis de deficiência visual e 62 crianças videntes, entre 15 e 61 meses de idade. Os resultados indicaram que não houve diferença significativa entre os grupos no que se refere a alguns fatores investigados quanto ao comportamento parental (nos fatores afeto e responsividade). Quanto à qualidade e à adequação de fatores investigados no envolvimento pai-mãe/cuidador (envolvimento físico e verbal, diretividade), também não ocorreram diferenças significativas. No entanto, o grupo das mães das crianças com deficiência visual mostrou maior envolvimento físico e controle da atividade, além de maior envolvimento verbal, apesar de a diferença neste último fator não ter sido estatisticamente significativa. 
Outro aspecto ressaltado por Behl et al. (1996) é que o fato de a mãe ter um filho com DV não significa necessariamente que o estilo interacional materno se mostre deficiente. Os autores consideram que as mães de crianças com deficiência visual demonstram maior nível de comportamentos diretivos, mas, ainda assim, demonstram também responsividade e afeto apropriados. Possivelmente as diferenças encontradas sejam um reflexo das tentativas das mães de usarem vias alternativas à visão para entrarem em contato com a criança. Este estudo contrasta com estudos anteriores (Fraiberg, 1977; van Hasselt, Hersen, Moor \& Simon, 1986), cuja premissa era de que a presença de uma deficiência visual na criança resultaria em possíveis distúrbios no sistema relacional mãe-criança. Finalmente, Behl et al. (1996) afirmam que os achados podem refletir um resultado positivo em relação à intervenção precoce, uma vez que todos os participantes frequentavam programas de intervenção oferecidos nos Estados Unidos na década de 1990, os quais eram praticamente inexistentes em décadas anteriores.

Resultados nesta mesma direção foram demonstrados por Campbell (2003) ao realizar um estudo comparativo sobre a diretividade materna em um contexto de brincadeira livre entre a mãe e a criança. Participaram do estudo um grupo de quatro crianças totalmente cegas, sem outras deficiências, e um grupo controle de quatro crianças com desenvolvimento típico, com 18 meses de idade. Uma vez que há divergências entre autores quanto ao conceito de diretividade, foram utilizadas cinco categorias de diferentes tipos de diretividade. Os resultados mostraram que não houve diferenças significativas quanto à diretividade materna, de modo geral. As mães das crianças com deficiência visual fizeram um maior número de tentativas de interromper um comportamento de risco para a criança, ou seja, diretividade negativa (ex.: pediam para as crianças não esfregarem os olhos). Por último, apesar de ter ocorrido uma tendência entre as mães das crianças com deficiência visual de solicitar alguma atividade, a diferença entre os grupos não foi significativa. As autoras indicam que as diferenças que apareceram mostram uma adequação das mães às necessidades das crianças, afirmando que nem sempre a diretividade está relacionada a uma falta de sensibilidade materna.

540 Conclusão semelhante foi apresentada por Sigolo (2000) ao chamar a atenção para a controvérsia sobre os benefícios/prejuízos da diretividade materna em populações atípicas.

Ao apresentarem a perspectiva desenvolvimental intersubjetiva, que envolve compreender o mundo subjetivo da criança (compartilhado com o cuidador) ao invés de compreendê-lo a partir de seu desenvolvimento individual, Loots, Devisé e Sermijn (2003) indicam os possíveis prejuízos no desenvolvi-mento da intersubjetividade entre mães e filhos com DV. Para eles, a intersubjetividade emergente e a física parecem estar em risco, uma vez que as mães e os bebês têm que enfrentar o desafio de reconhecer os convites um do outro para estabelecer e manter um ciclo de interação de comportamentos, atividades e afeto sincronizados. Como a intersubjetividade existencial se expande a partir das interações recíprocas e antecipações dos comportamentos da criança e do outro, dando uma consciência da interação para a criança, a possibilidade de compartilhar com o outro seus afetos e intenções também pode estar em risco nas crianças com deficiência visual. Loots et al. (2003), da mesma forma que Souza et al. (2005) e Pérez-Pereira e Contí-Ramsden (1999), sugerem que as mães das crianças com DV devam ser encorajadas a utilizar vias alternativas à visual para desenvolver a intersubjetividade, o que requer uma eficiente intervenção precoce.

\section{Intervenção precoce em crianças com deficiência visual}

O termo intervenção precoce, segundo Guralnick (1997), vem sendo utilizado para aquelas ações que buscam minimizar, o mais cedo possível, o impacto frente à criança com deficiência ou frente a situações de risco, uma vez que os primeiros anos de vida têm sido considerados um período sensível.

Atualmente, há consenso sobre quais características de programas de intervenção precoce tendem a ser mais efetivas. Uma delas é a importância de centrar a intervenção nas necessidades da família. Outros fatores fundamentais são a oferta de programas na própria comunidade, a integração de diferentes áreas do conhecimento e a implementação de uma rede de serviços de apoio (Callias, 1994; Guralnick, 1997). Tem sido apontada a importância de uma estrutura apropriada, contin- 
gente, encorajadora, afetiva, não intrusiva, baseada no diálogo e na interação cuidador-criança e com um padrão de sensibilidade aos aspectos desenvolvimentais para otimizar o desenvolvimento infantil adequado (Guralnick, 1997).

No entanto, no que se refere às díades mãe-bebê com deficiência visual, poucos estudos têm se dedicado a investigar a efetividade de intervenções. Um fato que pode explicar o reduzido número de pesquisas com intervenções nessa população é a complexidade metodológica envolvida nessa temática. A população de crianças com deficiência visual é extremamente heterogênea. As causas da deficiência são múltiplas e trazem importantes diferenças entre essas crianças, e cada uma dessas situações traz peculiaridades para o contexto interacional. Essa questão tem dificultado pesquisas que envolvam um grupo maior de crianças e um emparelhamento metodológico adequado, havendo mais investigações que empregam estudos de caso.

Em função dessa peculiaridade da população estudada, Warren (1994) ressaltou a necessidade de utilização de uma "abordagem individual das diferenças". Nessa abordagem, distinta da comparativa, o objetivo não é comparar diferentes populações entre elas, mas sim explicar diferenças dentro de uma população. A abordagem levanta uma questão de âmbito maior: qual é a natureza e quais são as causas das variações dentro da população estudada? Desta maneira, Warren interessa-se mais por aquilo que se pode realizar para uma meta de desenvolvimento possível dentro de um determinado grupo. Um primeiro passo nesta abordagem é uma descrição cuidadosa das características da população estudada, que envolva não somente médias, mas os extremos dentro desta população. Identificar as relações e, se possível, as causas das variações entre os indivíduos com deficiência visual seria um segundo passo importante, apontado pelo autor.

Ainda que haja poucos estudos, Aitken (1988) ressalta a importância da investigação acerca da efetividade da intervenção precoce. O autor ressalta que, quanto mais precoce for a intervenção, melhor será seu efeito. Infelizmente, as pesquisas que têm sido realizadas com essa população não têm mostrado resultados conclusivos, em função da falta de estudos bem controlados.
Um estudo que trouxe contribuições foi o de Adelson e Fraiberg (1974). As autoras realizaram uma intervenção cujo objetivo era melhorar a locomoção da criança, por iniciativa própria, a partir do desenvolvimento da coordenação som/mão (toque). 0 objetivo era auxiliar a criança a identificar o som como um estímulo que lhe chamasse a atenção, da mesma forma que o estímulo visual funciona para as crianças videntes. Para alcançar esse objetivo, a intervenção envolveu os pais em observações e orientações, pois, para as autoras, o parceiro humano do bebê é que lhe dá a maioria dos significados para toda a experiência sensorial. Desta forma, a intervenção centrou-se na relação pais-bebê e envolvia visitas quinzenais à família. Os participantes da pesquisa eram dez bebês cegos, sem outras deficiências, que tinham entre um e 11 meses de idade. Os dados do grupo controle foram oriundos de outro estudo.

As crianças foram avaliadas, mensalmente, por meio da Escala de Desenvolvimento Bayley, em relação ao seu desenvolvimento motor, para verificar a evolução da intervenção. Os resultados das avaliações foram comparados aos de um estudo anterior. As autoras apontaram que a intervenção trouxe efeitos positivos, possibilitando um desenvolvimento mais próximo ao das crianças videntes naqueles indivíduos que participaram da intervenção. Além disso, as autoras confirmaram a hipótese inicial de que a DV tem pouco impacto na aquisição dos itens posturais, apesar de estar associada a um marcado atraso no alcance de habilidades que envolvem mobilidade com iniciativa própria.

Klein, van Hasselt, Trefelner, Sandstrom e Brandt-Snyder (1988) realizaram um estudo com um amplo programa de intervenção multiprofissional, que envolvia encontros grupais com os pais dos bebês, entrevistas individuais e visitas à casa das famílias, enfocando o desenvolvimento das crianças com DV. Embora o programa tenha sido avaliado positivamente pelos participantes, existe a ressalva de que não foi realizado um delineamento experimental ou um estudo de caso aprofundado que pudesse indicar a efetividade da intervenção no desenvolvimento das crianças.

Hall e Bailey (1989) propuseram um modelo de intervenção com foco na visão funcional para crianças com baixa visão. O programa de treinamento das habilidades da criança era baseado na performance visual da 
mesma, avaliada por meio de exames oftalmológicos, optométricos, de baixa visão e de visão funcional. Esta última levou em consideração aspectos relevantes da observação dos pais e de outras pessoas significativas na vida da criança, assim como observações em setting natural. As autoras determinavam quais habilidades cada criança poderia provavelmente adquirir, quais necessitavam ser refinadas ou quais as crianças necessitavam adaptar a novas tarefas e situações, embora já as houvessem adquirido.

Lueck, Dornbusch e Hart (1999) avaliaram uma intervenção em três crianças com DV cortical, entre um e três anos de idade. Contudo, neste artigo reportaram somente os resultados da avaliação de uma intervenção com foco na visão funcional em uma criança de 14 meses com DV cortical. As autoras enfatizaram as dificuldades metodológicas envolvidas no estudo, uma vez que a população com essa deficiência é rara e muito diversa; a amostra não pôde ser pareada e estudos comparativos tornaram-se impossíveis. Portanto, foi utilizado o método de estudo de caso único para cada criança do estudo, envolvendo medidas de pré e pós-intervenção. Os efeitos de maturação foram controlados, uma vez que a intervenção tinha a duração de apenas 15 semanas. A intervenção enfatizava a necessidade de avaliar cada caso e oportunizar atividades que se enquadrassem com as necessidades da criança.

O estudo de Lueck et al. (1999) mostrou uma evolução positiva da criança e também apontou a necessidade de a intervenção se estender por mais tempo. Contudo, uma intervenção mais longa apresentaria dificuldades para avaliação de sua efetividade, uma vez que se tornaria difícil separar quais evoluções da criança se devem à intervenção ou à maturação. No entanto, o estudo sugere que, clinicamente, são necessárias intervenções mais prolongadas, que possam continuar motivando um melhor desenvolvimento da visão funcional. Um ponto a ser ressaltado na intervenção proposta pelas autoras é a inclusão das atividades delineadas na rotina da díade mãe-bebê, tendo sido a própria mãe quem realizava as atividades e pontuava os acertos da criança. As pesquisadoras realizavam o acompanhamento semanal dessas atividades. O estudo demonstrou que essa forma de intervenção motivou a mãe a interagir mais com seu bebê, podendo reconhecer

542 o potencial de aprendizagem do filho e estimulá-lo de forma mais adequada em outras ocasiões, além daquela proposta pela intervenção. Outro fator que as autoras discutem é um possível efeito da idade da criança ao participar da intervenção, uma vez que essa criança relatada no estudo teve sucesso em sua evolução e uma outra criança, de três anos de idade (na qual também foi aplicada a mesma intervenção), não evoluiu tão satisfatoriamente no mesmo período do treinamento. Esse resultado parece apontar para a importância da intervenção o mais precoce possível e para os fatores que promovem ou dificultam o envolvimento familiar nos programas.

A partir da revisão da literatura, pode-se afirmar que os estudos sobre intervenção precoce com crianças com DV são raros. Visando contribuir para delinear intervenções com essa população, serão discutidos alguns aspectos essenciais para a realização de intervenção precoce.

Guralnick (1997) propõe um modelo teórico que inter-relaciona o desenvolvimento infantil com a estrutura familiar e os possíveis fatores estressores nesse contexto. Para o autor, o desenvolvimento infantil resultará de alguns padrões familiares, tais como a qualidade da interação pais-filhos, as condições da família em oferecer novas experiências e novos estímulos para a criança e a possibilidade de a família prover saúde e segurança ao filho. Esses padrões familiares sofrem influências de acordo com as características pessoais dos pais, como grau de depressão, nível educacional, experiências parentais de gerações anteriores, expectativas culturais, assim como com as características não relacionadas com a deficiência ou com o risco biológico, tais como apoio social, relação conjugal, recursos financeiros ou temperamento infantil.

Além dos fatores acima descritos, Guralnick (1997) enfatiza que a família também enfrenta a presença de fatores potencialmente estressores interligados à deficiência do filho ou a um risco biológico. Primeiramente, o autor cita a necessidade de informações dos pais em relação à saúde e ao desenvolvimento do filho, incluindo suas crenças sobre o comportamento do filho, dúvidas sobre o manejo com a criança e o impacto do diagnóstico nas expectativas sobre o desenvolvimento do filho.

Um segundo ponto referido pelo autor é o estresse familiar e interpessoal que pode ocorrer. Essa 
vivência de estresse tem potencial para afetar a interação conjugal e parental. Outro aspecto enfatizado por Guralnick (1997) como potencialmente estressor é a necessidade de busca de recursos, sendo difícil para a família dar conta de tantas obrigações e responsabilidades. Ao mesmo tempo, muitas vezes os serviços não respondem adequadamente às necessidades da família em proporcionar a melhor terapêutica, oportunidades de educação e experiências sociais para o filho.

Por fim, um quarto aspecto referido pelo autor é a sensação de impotência que a família sente frente aos perigos relacionados à deficiência ou risco biológico que a criança apresenta. A falta de confiança e convicção da família em seu próprio potencial para auxiliar o filho pode minimizar a habilidade para solucionar os problemas que surgem (Schmidt \& Bosa, 2003). Manter a família confiando em seu potencial e no seu próprio poder de controle de decisões é essencial para o seu bem-estar e o da criança por um longo período (McDaniel, Hepworth \&Doherty, 1994).

Naturalmente, cada família tem uma distinta combinação dos diversos fatores envolvidos, e cada aspecto se caracteriza por uma intensidade diferente. Atentar para as necessidades individuais de cada família torna-se essencial para a efetividade de uma intervenção (Callias, 1994).

Ferrel (1984) cita dificuldades encontradas pelas famílias, as quais devem ser levadas em conta ao se elaborar um programa de intervenção. Pais de crianças com DV relataram passar por diversas dificuldades no que se refere ao apoio de profissionais. O fato de haver poucos estudos que digam respeito ao desenvolvimento inicial desta população dificulta que os profissionais tenham clareza para explicar a temática. Portanto, dúvidas a respeito das áreas que podem sofrer atrasos e formas de intervir para evitar problemas comuns são constantemente relatadas pelos pais.

Os pais referiram ainda ser comum a ênfase de profissionais em ensiná-los a lidar com os filhos como se fossem professores ou terapeutas, em detrimento das suas necessidades emocionais. Por outro lado, Lueck (2004) relatou a importância de se incluírem tarefas que abranjam diversas habilidades da criança em intervenções que auxiliem no desenvolvimento e, consequentemente, no acesso das crianças à escolarização e à integração psicossocial. Pode-se pensar que uma intervenção efetiva seria aquela que pudesse apoiar esses pais a serem pais e não professores, ao mesmo tempo em que habilitasse os pais a realizarem as tarefas necessárias de ensino que uma criança com deficiência demanda.

\section{Considerações Finais}

São poucos os estudos que investigam a efetividade de programas de intervenção precoce entre bebês com DV, pois a maioria focaliza o desenvolvimento das crianças entre dois e seis anos ou mais. Além disso, são escassas as informações a respeito do desenvolvimento das crianças dessa faixa etária, nesta área.

O fato de a população ser extremamente heterogênea, envolvendo, além da DV, outras deficiências associadas, dificulta a investigação de um número maior de participantes. Em função disso, poucos estudos investigam a efetividade da intervenção. Ainda que estudos de eficácia sejam mais difíceis de serem realizados nessa população, investigações de efetividade são possíveis, apesar de não seguirem os mesmos critérios rigorosos dos estudos de eficácia.

Ressalta-se, portanto, a necessidade de estudos longitudinais sobre o desenvolvimento inicial de crianças com deficiência visual, com o intuito de coletar informações que auxiliem os pais a redimensionarem suas expectativas em relação ao desenvolvimento de seus filhos. Além disso, é urgente que se investigue a efetividade de intervenções em fases tão iniciais do desenvolvimento, com o intuito de possibilitar um melhor atendimento para bebês com DV e suas famílias.

\section{Referências}

Adelson, E., \& Fraiberg, S. (1974). Gross motor development in infants blind from birth. Child Development, 45, 114-126.

Aitken, S. (1988). Whither early intervention? Retrieved November 9, 2004, from www.visugate.biz/bjvi/1988/ summer 1988.html

Als, H., Tronick, E., \& Brazelton, T. B. (1980). Stages of early behavioral organization: the study of a sighted infant and a blind infant in interaction with their mothers. In T. M. Field (Org.), High riskinfants and children, adult and peer interaction (pp.181-204). New York: Academic Press.

Batista, C., \& Enumo, S. (2000). Desenvolvimento humano e impedimentos de origem orgânica: o caso da deficiência 
visual. In H. A. Novo \& M. C. Menandro (Orgs.), Olhares diversos: estudando o desenvolvimento humano (pp.157-174). Vitória: UFES.

Behl, D., Akers, G., Boyce, M., \& Taylor. (1996). Do mothers interact differently with children who are visually impaired? Journal of Visual Impairment \& Blindness, 90 (6), 501-511.

Bosa, C. A. (2002). Atenção compartilhada e identificação precoce do autismo. Psicologia: Reflexão e Crítica, 15 (1), 77-88.

Callias, M. (1994). Parent training. In M. Rutter, E. Taylor \& L. Hersov. Child and adolescent psychiatry:modern aproaches (pp.918-935). Oxford: Blackwell Science.

Campbell, J. (2003). Maternal directives to young children who are blind. Journal of Visual Impairment \& Blindness, 97 (6), 355-365.

Ferrel, K. (1984). Early years action plan. Journal of Visual Impairment \& Blindness, 78, 56-57.

Fraiberg, S. (1977). Insights from the blind:comparative studies of blind and sighted infants. New York: Basic Books.

Gilbert, C, \& Foster, A. (2001). Childhood blindness in the context of vision 2020: the right to sight. Bulletin of the World Health Organization, 79 (3), 227-232.

Guralnick, M. (1997). The effectiveness of early intervention. Baltimore: Paul H. Brookes Publishing.

Hall, A., \& Bailey, I. L. (1989). A model for training vision functioning. Journal of Visual Impairment \& Blindness, 83 (8), 390-396.

Kekelis, L., \& Andersen, E. (1984) Family communication styles and language development. Journal of Visual Impairment \& Blindness, 78, 54-65.

Klein, B., van Hasselt, V. B., Trefelner, M., Sandstrom, D. J., \& Brandt-Snyder, P. (1988). The parent and toddler training project (PATT) for visually impaired and blind multihandicapped children. Journal of Visual Impairment \& Blindness, 82 (2), 59-64.

Loots, G., Devisé, I., \& Sermijn, J. (2003). The interaction between mothers and their visually impaired infants: an intersubjective developmental perspective. Journal of Visual Impairment \& Blindness, 97 (7), 403-417.
Lueck, A. H. (2004). Relating functional vision assessment, intervention, and outcomes for students with low vision. Visual Impairment Research, 6 (1), 45-52.

Lueck, A., Dornbusch, H., \& Hart, J. (1999). The effects of training on a young child with cortical visual impairment: an exploratory study. Journal of Visual Impairment \& Blindness, 93 (12), 778-793.

McDaniel, S. H., Hepworth, J., \& Doherty, W. J. (1994). Terapia familiar médica: um enfoque biopsicossocial às famílias com problemas de saúde. Porto Alegre: Artes Médicas.

Messer, D. (1994). The development of communication: from social interaction to language. Chichester, UK: John Wiley \& Sons.

Pérez-Pereira, M., \& Contí-Ramsden, G. (1999). Language development and social interaction in blind children. Eas Sussex, UK: Psychology Press.

Rogers, S., \& Puchalsky, C. B. (1984). Social characteristics of visually impaired infant's play. Topics in Early Childhood Special Education, 3 (4), 52-56.

Rowland, C. (1984). Preverbal communication of blind infants and their mothers. Journal of Visual Impairment \& Blindness, 78 (7), 297-302.

Schmidt, C., \& Bosa, C. (2003). A investigação do impacto do autismo na família: revisão crítica da literatura e proposta de um novo modelo. Interação, 7 (2), 111-120.

Souza, A. D., Bosa, C., \& Hugo, C. N. (2005). As relações entre deficiência visual congênita, condutas do espectro do autisto e estilo materno de interação. Estudos de Psicologia (Campinas), 22 (4), 355-364.

van Hasselt, V., Hersen, M., Moor, L., \& Simon, J. (1986). Assessment and treatment of families with visually handicapped children: a project description. Journal of Visual Impairment \& Blindness, 80 (3), 633-635.

Warren, D. H. (1994). Blindness and children: an individual differences approach. London: Cambridge University Press.

Recebido em: 28/6/2007

Versão final reapresentada em: 17/1/2008

Aprovado em: 11/3/2008 